
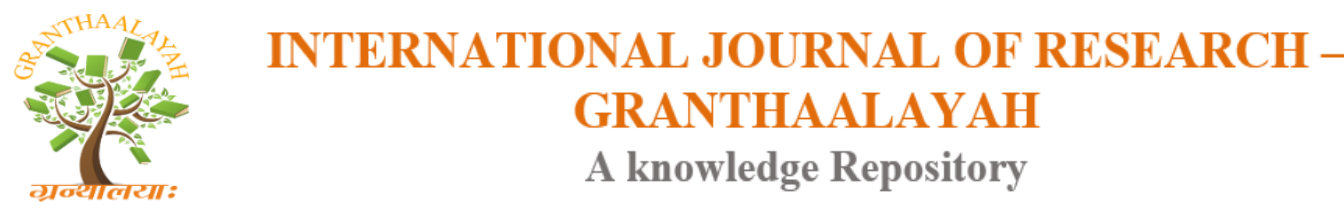

Science

\title{
SYNCHRONIZATION IN RELATIVITY IN THE CONTEXT OF THE TWIN PARADOX
}

\author{
Chandru Iyer ${ }^{* 1}$ \\ ${ }^{* 1}$ Plant Head, Lydall Performance Materials, Sohna Plant, Sohna 122103, Haryana, India
}

\begin{abstract}
We present a common understanding of clock synchronization as opposed to the synchronization convention adopted by SRT in the context of the Twin Paradox; we place two spatially separated observers in the stationary and travelling frames and discuss the synchronization issues at the completion of the travels.

Keywords: Synchronization; Relativity; Context; Twin Paradox.

Cite This Article: Chandru Iyer. (2018). "SYNCHRONIZATION IN RELATIVITY IN THE CONTEXT OF THE TWIN PARADOX." International Journal of Research - Granthaalayah, 6(9), 274-277. https://doi.org/10.5281/zenodo.1443437.
\end{abstract}

\section{Introduction}

The twin paradox has been a widely discussed topic for over hundred years. Students and Physicists alike continue to discuss the problem [1 to 15] with profound interest. The main reason for this continuing discussion is that according to SRT, the two clocks ('travelling' and 'stationary') are equivalent in principle as long as they remain in their respective inertial frames and no acceleration is induced or experienced. This leads to the surmising that the so-called youngness of the traveller Stella, could not have happened during her travels but only when she turned around to return. But this poses some problems as the turning around can be visualized as close to a instantaneous process and not linearly or proportionally related with the amount of distance/ time travelled by Stella, whereas the quantum of youngness of Stella compared to the 'stationary' Eartha is linearly and proportionally related to the distance/ time spent by Stella in her travels. In common experiences also we do experience sharp turnarounds (U urn) of vehicles, walkers and joggers and we are able to visualize a close to instantaneous turn around and so it is difficult to imagine how a substantial difference in ageing can possibly happen during such a turnaround. In conjunction with the surmise that Stella could not have become younger during the periods of travel when she was in uniform motion with respect to Eartha, the question still remains to be answered as to "during what intervals of time Stella actually became younger". 
This problem is closely related to the synchronization convention adopted by SRT in inertial frames and we shall present a situation highlighting the issues relating to synchronization as ordained by SRT and the common sense understanding of the same concept of synchronization.

\section{Concept of Synchronization in SRT and Common Sense}

We discuss the concept of synchronization as envisaged by SRT and as understood in common parlance.

Concept of Synchronization in SRT: The synchronization of two clocks in SRT is possible only when they are continuously present in the same inertial frame. This synchronization is achieved by assuming the constancy of the one way speed of light. This synchronization is identical to the synchronization achieved by slowly separating two synchronized clocks initially present at the same location in the same inertial frame.

Concept of Synchronization in Common Parlance: The meaning of synchronization, according to common understanding, is that two identical clocks synchronized when they are at the same location, will show the same time when they meet again provided they go through the same physical movements and processes.

It is conceivable that two clocks can be synchronized by synchronizing each of them with a third clock. However, when the three clocks are in uniform motion with respect to each other, the formulations of SRT, ordain otherwise. We have discussed this in detail in an earlier article [14]. To recollect, in this paper we describe two clocks $\mathrm{k}, \mathrm{m}$ moving at speeds $\mathrm{v}, \mathrm{u}$ (such that $\mathrm{v}>\mathrm{u}$ ) with respect to a clock $\mathrm{n}$ ( $\mathrm{n}$ being considered stationary). The three clocks $\mathrm{k}, \mathrm{m}$ and $\mathrm{n}$ meet each other on three occasions in pairs (event E1 clock k passing clock m, event E2 clock k passing clock n, event $\mathrm{E} 3$ clock $m$ passing clock $n$, in that time order) and yet they could not synchronize themselves (according to SRT). In normal understanding of synchronization, these three clocks should have been able to synchronize themselves after the three paired meetings.

We have discussed the importance of synchronization in inertial frames, in the context of slowing down of time in moving clocks, in an earlier article [15]. It is widely recognized that the apparent slow running of moving clocks is a result of the different synchronizations adopted by the inertial frames. We discuss the SRT synchronization convention in the context of the common understanding of synchronization outlined in the previous paragraph by a situation visualized in the next section.

\section{Quadruplet Paradox}

Units of distance $=$ Light Years

Units of time $=$ Years

Speed of light $=1$

One dimensional space

Let us say two observers A and B are located 0.4 light years apart in an inertial frame K. At the instant $\mathrm{t}=0$ in $\mathrm{K}$, observers $\mathrm{P}$ and $\mathrm{Q}$ in inertial frame $\mathrm{K}$ ', moving at $\mathrm{v}=0.8$ light years / year are 
crossing $\mathrm{A}$ and $\mathrm{B}$ respectively. $\mathrm{P}$ is passing $\mathrm{A}$ and $\mathrm{Q}$ is passing $\mathrm{B}$ at $\mathrm{t}=0$ in $\mathrm{K}$. At this instant in $\mathrm{K}$, coordinates of $\mathrm{A}$ are $(\mathrm{x}=0 ; \mathrm{t}=0)$ and coordinates of $\mathrm{B}$ are $(\mathrm{x}=-0.4 ; \mathrm{t}=0)$

Let us design the experiment such that P's clock is synchronized with A's as they cross each other and therefore $\mathrm{P}$ 's clock shows $\mathrm{t}^{\prime}=0$ as it crosses $\mathrm{A}$. The clock of $\mathrm{Q}$ is synchronized with $\mathrm{P}$ as per the SRT convention and therefore, will show a time of $-\mathrm{vx} \gamma / \mathrm{c}^{2}=-(0.8)(-0.4)(5 / 3)=(8 / 15)$

Let us say P and Q both travel for 5 years (as observed by K that is by observers $\mathrm{A}$ and B) and they both turn around and travel at $\mathrm{v}=-0.8$ and reach the same location after 5 more years and pass $\mathrm{A}$ and $\mathrm{B}$ again.

The passing of $\mathrm{A}$ and $\mathrm{P}$ are observed by $\mathrm{A}$ at $\mathrm{x}=0 ; \mathrm{t}=10$

As per the well accepted principle of the less ageing of the travelling $\mathrm{P}$, the clock of $\mathrm{P}$ will show $\mathrm{t}^{\prime}=6$ as it passes $\mathrm{A}$.

Similarly the passing of $\mathrm{Q}$ and $\mathrm{B}$ will be observed at $\mathrm{x}=-0.4 ; \mathrm{t}=10$ by $\mathrm{B}$ and the clock of $\mathrm{Q}$ will show $6+(8 / 15)$ as $\mathrm{Q}$ had a time of $(8 / 15)$ when it left $\mathrm{B}$ at $\mathrm{t}=0$.

Now the asynchronization between $\mathrm{P}$ and $\mathrm{Q}$ as observed by $\mathrm{K}$ is $8 / 15$.

As per SRT, the (revised) asynchronization between P and Q should be -vx $\gamma / \mathrm{c}^{2}=-(-0.8)(-0.4)$ $(5 / 3)=-8 / 15$

This means the two clocks P and Q have lost their synchronization according to SRT. Whereas the clocks $\mathrm{P}$ and $\mathrm{Q}$ have gone through the same physical processes and movements and they were initially synchronized in accordance with the accepted synchronization convention of SRT.

4.0 Conclusion: Whereas there is no conceivable reason for the clocks $\mathrm{P}$ and $\mathrm{Q}$ which were originally synchronized, to lose their synchronization during the identical movements and acceleration as described in section 3, according to SRT, P and Q lost their synchronization by an amount 16/15 years. This shows there is a conceptual difference between the common parlance understanding of synchronization and the synchronization defined by SRT.

Whereas SRT determines that P and Q have lost their synchronization and need to re-synchronize, there should be no need for such re-synchroniztion in common sense understanding of synchronization as $\mathrm{P}$ and $\mathrm{Q}$ have gone through the same movements and processes. This gets further emphasized by the fact that according to SRT, bringing P and Q together slowly will show that they were synchronized (in the beginning) and the same process of checking synchronicity (bringing $\mathrm{P}$ and $\mathrm{Q}$ together slowly) will show that $\mathrm{P}$ and $\mathrm{Q}$ have lost there synchronicity when they crossed A and B for the second time. This loss of synchronization between $\mathrm{P}$ and $\mathrm{Q}$ as determined by SRT is not dependent on any particular observing frame of reference.

\section{References}

[1] Romer R H 1959, "Twin paradox in special relativity," Am. J. Phys. 27, 131-135.

[2] Bohm D 1965, The Special Theory of Relativity, (W. A. Benjamin, New York), 165-172. 
[3] Taylor E F and Wheeler J A 1992 Spacetime Physics, $2^{\text {nd }}$ Edition (W. H. Freeman, New York), 7677.

[4] Langevin P 1911 "L'évolution de l'espace et du temps," Scientia X, 31-54.

[5] Debs T A and Redhead M L G 1995 "The twin "paradox' and the conventionality of simultaneity," Am. J. Phys. 64, 384-392.

[6] Einstein A 1918, "Dialog über Einwände gegen die Relativitätstheorie," Die Naturwissenschaften 48, 697-702. (English Translation available in The Collected Papers of Albert Einstein, Volume 7, The Berlin Years: Writings, 1918-1921, Princeton Univ. Press, Princeton, New Jersey, 2002, pp. 66-75).

[7] Unruh W G 1981 "Parallax distance, time, and the twin paradox," Am.J. Phys. 49, 589-592.

[8] Nikolic H 1999 "Relativistic contraction of an accelerated rod," Am. J. Phys. 67, 1007-1017.

[9] Good R H 1982 "Uniformly accelerated reference frame and twin paradox," Am. J. Phys. 50, 232238.

[10] Boughn S P 1989 “The case of the identically accelerated twins," Am. J. Phys. 57, 791-793.

[11] Perrin R 1979 "Twin paradox: a complete treatment from the point of view of each twin," Am. J. Phys. 47, 317-319.

[12] Grøn Ø 2006 "The twin paradox in the theory of relativity," Eur. J. Phys. 27, 885-889.

[13] Low R J 1990 "An acceleration free version of the clock paradox," Eur. J. Phys. 11, 25-27.

[14] Iyer C, Prabhu G. M. 2007 Synchronizing equivalent clocks across inertial frames", arXiv 0710:1594

[15] Iyer C, Prabhu G.M. 2008 "A variation of the Clock Paradox and a distinguishing feature of an inertial frame", arXiv:0809.4465

\footnotetext{
*Corresponding author.

E-mail address: chandru_i@y ahoo.com
} 